\title{
EDITORIAL
}

\section{Was bleibt - von der deutschen Gegenwartsliteratur?}

Als einer der drei Herausgeber, Werner Jung, im akademischen Jahr 1999/ 2000 als Gastprofessor im German Department sowie innerhalb des >Literature Programs an der Duke University, NC Seminare und Vorlesungen zur Geschichte der Nachkriegsliteratur gehalten hat, dabei auf ein überaus reges Interesse unter Studierenden wie Lehrenden gestoßen ist, ist sehr schnell in Diskussionen mit dem Chair des German Department, James Rolleston, dem Acting Chair, Ann Marie Rasmussen, und - vor allem auch - den jüngeren Kolleg/innen die Idee zu einem Symposion entstanden, das nach dem >literarischen Feld < der deutschen Nachkriegs- resp. Gegenwartsliteratur, nach möglichen Periodisierungen in ihr und dem Problem des Bleibenden (auch und erst recht nach der $>$ Wende $<$ ), schließlich auch - unter Beteiligung einiger Autor/innen (Kerstin Hensel, Reinhard Jirgl, Angela Krauß, Silvia Szymanski) - nach der Aktualität der deutschen Literatur und ihren Spielräumen fragen sollte. Mit Ausnahme des (hier nachgelieferten) Essays von Martin Hielscher, der leider nicht am Symposion teilnehmen konnte, sind alle Beiträge während eines dreitägigen Symposions vom 29.-31.3.2001 vorgetragen worden und kommen hier - z. T. in überarbeiteter und erweiterter Form - zum Abdruck. Ganz besonderer Dank der Herausgeber gebührt an dieser Stelle noch einmal ausdrücklich James Rolleston, dessen unermüdlicher Ausdauer und Verhandlungsgeschick mit Sponsoren in erster Linie das Zustandekommen unseres Symposions zu verdanken ist. Die beiden anderen Herausgeber möchten sich darüber hinaus noch bei Mariatte Denmann bedanken, auf der - zu weiten Teilen - die logistische Last vor, während und auch nach Ablauf unseres Symposions gelegen hat. Last but not least gebührt der Duisburger Hilfskraft Alexandra Klein noch ein gehöriger Dank für die Einrichtung der Typoskripte.

Mariatte Denmann, Durham, NC; Peter McIsaac, Durham, NC; Werner Jung, Duisburg 